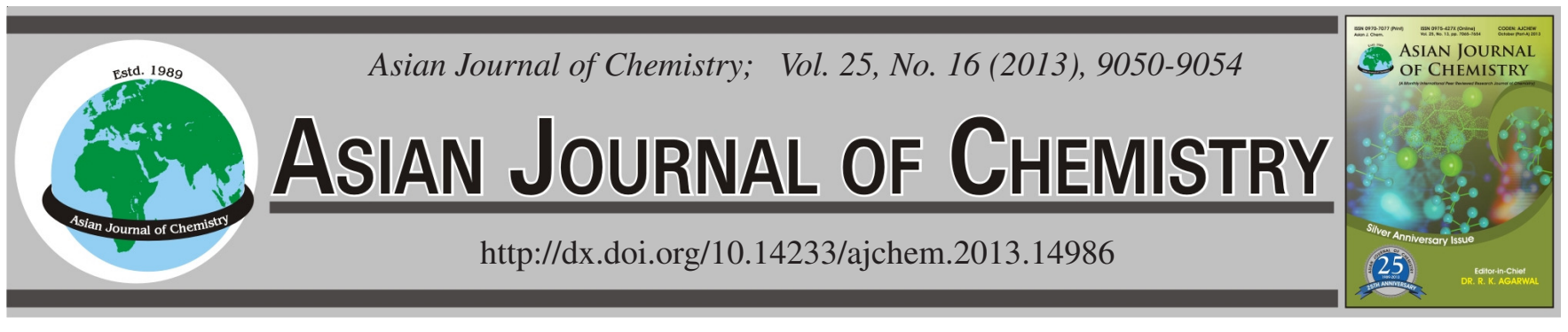

\title{
Selective Separation of L-Aromatic Amino Acid by L-Phenylalanine-Crosslinked Chitosan Resin
}

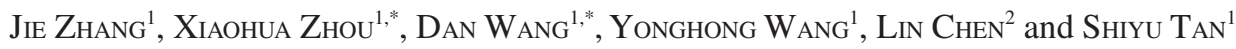

${ }^{1}$ College of Chemistry and Chemical Engineering, Chongqing University, Chongqing 400044, P.R. China

${ }^{2}$ Southwest Synthesis Pharmaceutical Corp., Ltd. Technology Center, Chongqing, P.R. China

*Corresponding authors: Tel./Fax: +86 23 65111179; E-mail: zhou65306590@hotmail.com; danwang088@gmail.com

\begin{abstract}
Selective separation of L-aromatic amino acid from amino acids mixture solution by L-phenylalanine-crosslinked chitosan resin (PCCR) was investigated in this study. L-Phenylalanine-crosslinked chitosan resin was successfully synthesized via the reaction between glutaraldehyde semi-crosslinked chitosan and L-phenylalanine. L-Phenylalanine-crosslinked chitosan resin could be considered as a macroporous anion exchanger with hydrophobic interaction. The $\pi$ - $\pi$ hydrophobic interaction was acted as an auxiliary function to improve the separation process. The optimum operating conditions were studied by batch adsorption and dynamic adsorption of L-phenylalanine on PCCR. The results were as following: the concentration of L-phenylalanine was $3 \mathrm{mg} / \mathrm{mL}, \mathrm{pH}$ was 6 and flow rate was $3 \mathrm{~mL} / \mathrm{min}$. L-Phenylalanine could be eluted effectively using $30 \%$ ethanol with $0.05 \mathrm{~mol} / \mathrm{L} \mathrm{NaOH}$ and flow rate was $0.5 \mathrm{~mL} / \mathrm{min}$. The results indicated that $\mathrm{L}-$ phenylalanine could be effectively separated from amino acids mixture by PCCR. The recovery rate of pure L-phenylalanine was $78 \%$. The hydrophobic interaction and electrostatic interaction between the L-phenylalanine and PCCR played an important role in the selective adsorption.
\end{abstract}

Key Words: Separation, L-Phenylalanine, Crosslinked chitosan, L-Aromatic amino acid.

\section{INTRODUCTION}

L-Aromatic amino acid is a kind of essential substance of body metabolism. It is widely applied in pharmaceutical, food and daily chemical industry. Although, the L-aromatic amino acid could be obtained by the chemical synthesis, its separation from fermentation liquid is more important.

At the present, the common method for separation of Laromatic amino acid from fermentation liquid is ion exchange ${ }^{1,2}$ or liquid-liquid extraction ${ }^{3-5}$. Ion exchange is based on the ionic bond. Liquid-liquid extraction is based on the electrostatic bond or hydrogen bond or coordination bond generated between the extractant and solute. Nevertheless, only part of L-aromatic amino acid could be separated by these two methods and the products are salt. In addition, the acids, alkalis and organics, which is achieved from recycle resin or extractant, cause environmental pollution. Therefore, it is important to research new materials and methods for the separation of L-aromatic amino acid.

L-Aromatic amino acid contains L-phenylalanine, Ltyrosine and L-tryptophan. The same characteristics of them are that the side chain is benzene ring or its derivatives. The benzene ring of L-aromatic amino acid has six coplanar carbon atoms and their ionic cloud, which is even distribution, could form conjugate double bond system. Theoretically, when a similar structure of L-aromatic amino acid is closed to the aromatic group, the $\pi$ - $\pi$ bonds of them can overlap partly or overall and the hydrophobic interaction ${ }^{6,7}$ could be formed based on the conjugated cyclic area between two similar cyclic structures. If the aromatic ring is used as the separation functional group and coupled it with the inert carrier, a new separation material could be obtained. The hydrophobic interaction between L-aromatic amino acid and separation material lays the basis for the efficient separation of L-aromatic amino acid.

In this study, L-phenylalanine-crosslinked chitosan resin (PCCR) used to separate L-aromatic amino acid was prepared in our laboratory, which was successfully synthesized via the reaction between glutaraldehyde semi-crosslinked chitosan and L-phenylalanine. It could be considered as a macroporous anion exchanger with hydrophobic interaction. The reaction progress was shown in Scheme-I. Selective adsorption of L-phenylalanine from mixed amino acid using PCCR as adsorbent was developed in this paper. The separation mechanism and process conditions of PCCR were examined in detail. 


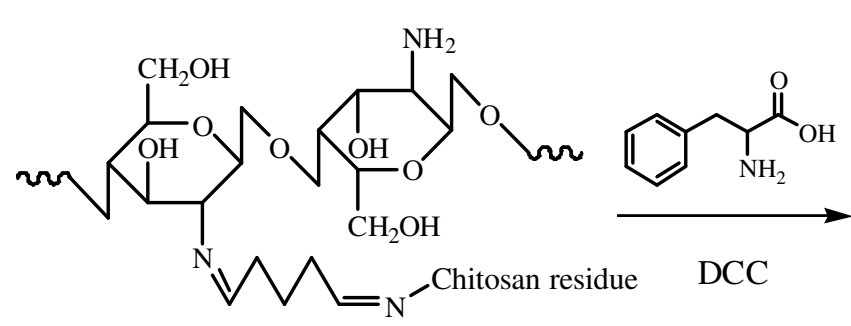

semi-clossinked chitosan

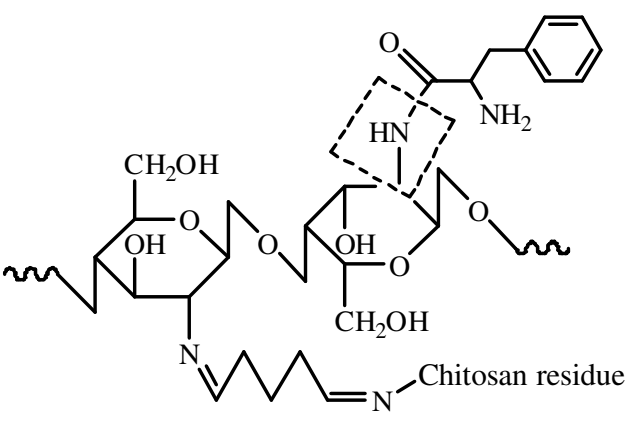

PCCR

Scheme-I: Synthesis reaction of semi-crosslinked chitosan and L-phenylalanine (DCC = N,N-dicyclohexylcarbodiimide)

\section{EXPERIMENTAL}

L-Phenylalanine (phe), L-tyrosine, alanine (ala), glutamate (glu), lysine (lys) and N,N-dicyclohexylcarbodiimide (DCC), were purchased from Qiude Biotechnology Co. (Shanghai, China). Chitosan (N-deacetylation degree, $95 \%$ ) were supplied by Yuhuan Biochemical Co. (Zhenjiang, China). Glutaraldehyde ( $25 \%, v / v)$, liquid paraffin, Tween-80, hydrochloride and other chemicals of analytical grade were purchased from Chongqing Chemical Head Factory (Chongqing, China). All chemicals were used without further purification unless otherwise specified. All aqueous solutions were prepared with double distilled water.

Preparation of L-phenylalanine-crosslinked chitosan resin: The semi-crosslinked chitosan resin $(\mathrm{CCR})^{8}$ were suspended in $200 \mathrm{~mL} 0.1 \mathrm{~mol} / \mathrm{L} \mathrm{L}$-phenylalanine solution and stirred moderately until adsorption equilibrium was approached. $30 \mathrm{~mL}$ ethanol $(95 \%, \mathrm{v} / \mathrm{v})$ mixed with $0.02 \mathrm{~mol} / \mathrm{L} \mathrm{N}, \mathrm{N}-$ dicyclohexylcarbodiimide was added to initiate the desired acylation reaction at room temperature for $1 \mathrm{~h}$ under mild agitation. At the end of reaction, the product was filtered under vacuum and washed with ethanol $(95 \%, \mathrm{v} / \mathrm{v})$ to remove the residual N,N-dicyclohexylcarbodiimide and its derivatives. Finally, the solid product was dialyzed with deionized water for $12 \mathrm{~h}$ to remove the residual L-phenylalanine or poly-(Lphenylalanine) and filtered again to obtain the final products (PCCR). The PCCR was stored at $4{ }^{\circ} \mathrm{C}$ for further study. Further more, the amino group of PCCR was blocked by ethyl bromide ${ }^{9}$ to achieve the inactive amino PCCR (IPCCR). At last, the recycle was necessary, the recycle method was as following: the desorption PCCR processed successively with $0.05 \mathrm{~mol} / \mathrm{L}$ $\mathrm{NaOH}$ ethanol $(60 \%)$ solution and $1 \% \mathrm{HCl}$. Finally, PCCR was washed by distilled water until neutrality.

Adsorption: Pure PCCR or IPCCR was suspended in amino acid solution for $1 \mathrm{~h}$ without stirring. The residual amino acids were assayed with the upper clear liquid on time to achieve the optimum condition. In another case, pure PCCR was packed into a glass chromatography column. The L-phenylalanine solution was pumped into the column and the L-phenylalanine concentration of effluent was determined by the UV detector automatically. Then the adsorption curve was drawn with the adsorption volume as the abscissa and the adsorption capacity of amino acid as the ordinate. And the adsorption capacity and the partition coefficient $\mathrm{K}$ were calculated by eqns. 1 and 2 .

$$
\begin{gathered}
\mathrm{Q}_{\mathrm{a}}=\frac{\mathrm{Co}_{\mathrm{o}}-\mathrm{m}}{\mathrm{W}_{\mathrm{p}}} \\
\mathrm{K}=\frac{\mathrm{Q}_{\mathrm{a}}}{\mathrm{Q}_{\mathrm{e}}}
\end{gathered}
$$

$\mathrm{Q}_{\mathrm{a}}(\mathrm{mg} / \mathrm{g})$ was the adsorption capacity, $\mathrm{C}_{0}(\mathrm{mg} / \mathrm{mL})$ and $\mathrm{V}_{0}$ $(\mathrm{mL})$ were the initial concentration and volume of amino acid in solution. $\mathrm{m}(\mathrm{mg})$ was the saturation adsorption capacity. $\mathrm{W}_{\mathrm{p}}$ was the weight of dry PCCR. $\mathrm{K}$ is the partition coefficient. $\mathrm{Q}_{\mathrm{e}}(\mathrm{mg} / \mathrm{g})$ was the elution capacity.

Elution: The eluting agent was pumped into the glass chromatography column which was after dynamic adsorption. The operation was also performed at the optimum condition determined by batch elution. Then the elution curve was drawn with the elution volume as the abscissa and the elution capacity of amino acid as the ordinate. The elution capacity was calculated by eqn. 3 .

$$
\mathrm{Q}_{\mathrm{e}}=\frac{\mathrm{m}}{\mathrm{W}_{\mathrm{p}}}
$$

Analysis of amino acid: The ninhydrin method ${ }^{10,11}$ was used to decide the concentrations of ala, glu, lys, L-phenylalanine and L-tyrosine in the static experiment. The concentration of L-phenylalanine in the dynamic experiment was decided by the UV spectrophotometer ${ }^{12}$.

\section{RESULTS AND DISCUSSION}

Selectivity of L-phenylalanine-crosslinked chitosan resin: In this work, four kinds of adsorbates standing for neutral, acidic, alkaline and aromatic amino, respectively, namely ala, glu, lys, L-phenylalanine and L-tyrosine were employed in this study to test the selective property of PCCR. There were obvious different results among CCR, IPCCR and PCCR, shown in Table-1. The adsorption capacity of L-phenylalanine on CCR, IPCCR and PCCR were 2.43, 0.45 and $2.70 \mathrm{mmol} / \mathrm{g}$. The adsorption capacity of PCCR was the highest.

The functional group of CCR was amino group. At $\mathrm{pH} 6$, those amino acids were complete negative except lysine, so the adsorption capacity was achieved only based on ion exchange. A $\beta$-phenyl group was introduced into PCCR, of which the adsorption capacity of L-phenylalanine and L-tyrosine, namely L-aromatic amino acid was enhanced. The experimental results showed PCCR had a better selective property to L-aromatic amino acid. 


\begin{tabular}{|c|c|c|c|c|c|}
\hline \multirow{4}{*}{ Resins } & \multicolumn{4}{|c|}{$\begin{array}{c}\text { TABLE-1 } \\
\text { STATIC ADSORPTION OF FOUR TYPES } \\
\text { AMINO ACIDS ON PCCR AND IPCCR }\end{array}$} & \\
\hline & \multicolumn{5}{|c|}{ Adsorption capacity (mmol/g dry resin) } \\
\hline & \multicolumn{5}{|c|}{ Amino acids } \\
\hline & L-Phenylalanine & L-Tyrosine & ala & glu & lys \\
\hline CCR & 2.43 & 2.41 & 2.71 & 2.16 & 1.34 \\
\hline IPCCR & 0.45 & 0.37 & 0.07 & 0.07 & 0.07 \\
\hline PCCR & 2.70 & 2.61 & 2.32 & 2.01 & 2.18 \\
\hline
\end{tabular}

Table-1 also illustrated that the adsorption capacity of different amino acid on IPCCR was different. The adsorption capacity of L-phenylalanine was the highest. Compared to glu and lys, the adsorption capacity of L-phenylalanine was improved $84.44 \%$. Recently, we had found ${ }^{13}$ that the selective adsorption was interacted with the hydrophobic interaction between two pyrrolidine rings. Based on the structure analysis of PCCR and IPCCR, it could be known that the functional groups of PCCR were ionization $\alpha$-amino group and $\beta$-phenyl group. It suggests that there were two interactions to separate amino acid. The electrostatic interaction formed between the $\alpha$-amino group of resin and the carboxyl group of amino acid. The $\beta$-phenyl group could form a hydrophobic region of six coplanar atoms enabling the benzene ring on amino acids to hydrophobically interact with the benzene ring on PCCR. Table-1 also showed that the average adsorption capacity of L-aromatic amino acid on PCCR was higher than the nonaromatic amino acid. And the added value was 0.42 . The added value was benefit from the hydrophobic interaction. Meanwhile, there was nonelectrostatic interaction between IPCCR and amino acid. L-Aromatic amino acids could be adsorbed by IPCCR via hydrophobic interaction. And the average added value was 0.41 . This result was similar to the added value of PCCR. So, it was proved that PCCR could separate L-aromatic amino acid selectively, based on the extra hydrophobic interaction between two large $\pi$ bonds. The separation mechanism was shown in Scheme-II.

Ala, glu and lys (Scheme-IIb) were only adsorbed by IPCCR with the hydrogen bond. Thus the adsorption capacity was the same. According to Scheme-IIa, L-tyrosine had a larger steric hindrance caused by hydroxyl, which leaded to a lower adsorption capacity than that of L-phenylalanine. PCCR had a better selectivity for L-phenylalanine. So, L-phenylalanine was taken to as an example to research the separation characteristics of PCCR on separation L-aromatic amino acid in this research.

Equilibrium time: The effect of equilibrium time on the adsorption of L-phenylalanine on PCCR was shown in Fig. 1. The shape of Fig. 1 showed that the adsorption of L-phenylalanine on PCCR was a typical Langmuir monolayer adsorption $^{14}$. The adsorption capacity was controlled by the area of inner channel. The adsorption reached equilibrium at $0.5 \mathrm{~h}$ and the highest adsorption capacity was $123.73 \mathrm{mg} / \mathrm{g}$. Generally, the adsorption equilibrium time of flavonoid on the macroporous resin was $1 \mathrm{~h}$ and the equilibrium adsorption capacity was $30-50 \mathrm{mg} / \mathrm{g}^{15,16}$. Whereas, the adsorption capacity of PCCR prepared in our laboratory was improved obviously with a short equilibrium time. That indicated that the porous

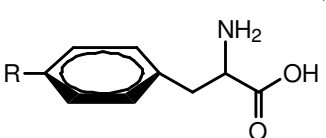<smiles>[R]C(N)C(=O)O</smiles>

$\mathrm{R}: \mathrm{H}-\mathrm{Phe}$
$\mathrm{OH}-\mathrm{Tyr}$

a
$\mathrm{R}: \mathrm{CH}_{3}-\mathrm{Ala}$

$\mathrm{C}_{2} \mathrm{H}_{4} \mathrm{COOH}-\mathrm{Glu}$

$\mathrm{C}_{4} \mathrm{H}_{8} \mathrm{NH}_{2}-$ Lys

b

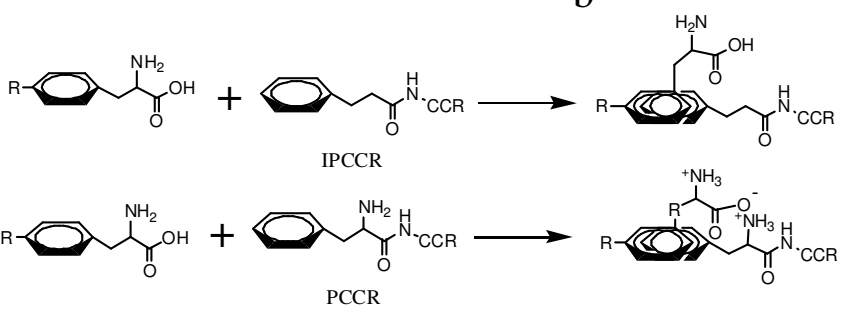

Scheme-II: Mechanism of the adsorption of L-phenylalanine, L-tyrosine, ala, glu and lys on PCCR

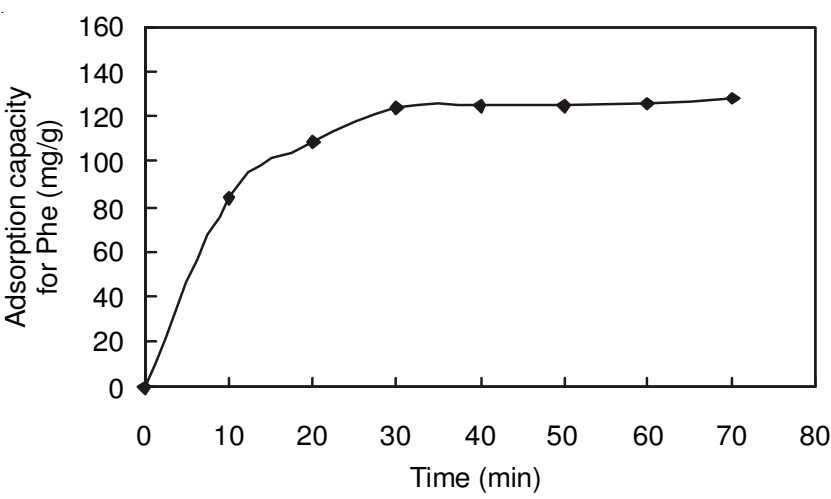

Fig. 1. Influence of time on the adsorption capacity of L-phenylalanine

structure of PCCR was beneficial for small molecules to transfer and adsorb.

pH: The effect of $\mathrm{pH}$ on the adsorption of L-phenylalanine on PCCR could be seen in Fig. 2. At pH 1-6, the adsorption capacity of L-phenylalanine on PCCR was increased with the increasing $\mathrm{pH}$. The separation of L-phenylalanine included two kinds of interaction, electrostatic interaction and hydrophobic interaction. The electrostatic interaction and ionization equilibrium of amino and carboxyl group were both effected by $\mathrm{pH}$. The $\mathrm{pK}$ value of amino group was closed to 10, (SchemeIII). Thus, the amino group was positive charged with $\mathrm{pH}$ ranging from 1-6. The $\mathrm{pK}$ value of carboxyl group was 2.2. With the increasing of $\mathrm{pH}$, carboxyl group was dissociated continually, which meant that the negative ion was increased. When $\mathrm{pH}$ was 6 , almost all the carboxyl group was negative. At this time, the electrostatic interaction achieved the maximum between the carboxyl group and amino group. So the adsorption capacity was the highest. When $\mathrm{pH}$ was higher than 6, the amount of protonated amino group was decreased gradually. The carboxyl group, which could form the electrostatic interaction, was decreased. So the adsorption capacity was decreased gradually. Especially when $\mathrm{pH}$ was more than 10, almost all of carboxyl group was nonprotonated and could not form the electrostatic interaction. The adsorption capacity kept invariant which was $73.5 \mathrm{mg} / \mathrm{g}$. This value was similar to the adsorption capacity of L-phenylalanine on IPCCR in Table-1. This result indicated that the $\pi-\pi$ hydrophobic interaction between PCCR 




Fig. 2. Influence of $\mathrm{pH}$ on the adsorption capacity of L-phenylalanine

$\overbrace{\mathrm{NH}_{3}{ }^{+}}^{\stackrel{\mathrm{OKKa}=2.2}{\rightleftharpoons}} \underbrace{\mathrm{O}}_{\mathrm{NH}_{3}{ }^{+}} \stackrel{\mathrm{OKa}_{2}=9.31}{=} \underbrace{\mathrm{O}}_{\mathrm{NH}_{2}}$

Scheme-III: Dissociation equilibrium of L-phenylalanine

and L-phenylalanine was acted as an important role to separate L-phenylalanine. Meanwhile, $\mathrm{pH}$ had no effect on the hydrophobic interaction $^{17-19}$.

Concentration of L-phenylalanine: The influence of L-phenylalanine concentration on adsorption capacity of L-phenylalanine on PCCR was studied. As it could be seen from Fig. 3, when the concentration was lower than $3 \mathrm{mg} / \mathrm{mL}$, the adsorption capacity of PCCR was increased with the increasing concentration of L-phenylalanine. After that, the adsorption capacity was kept stable at $122.65 \mathrm{mg} / \mathrm{g}$. This was mainly because the adsorption functional groups of pure PCCR were quantitative. Within a certain range, the adsorption probabilities increased between PCCR and L-phenylalanine with the increasing of solute concentration. Then the adsorption capacity was finally increased. As the functional groups of PCCR were saturated with excessive L-phenylalanine, the adsorption capacity was stable.

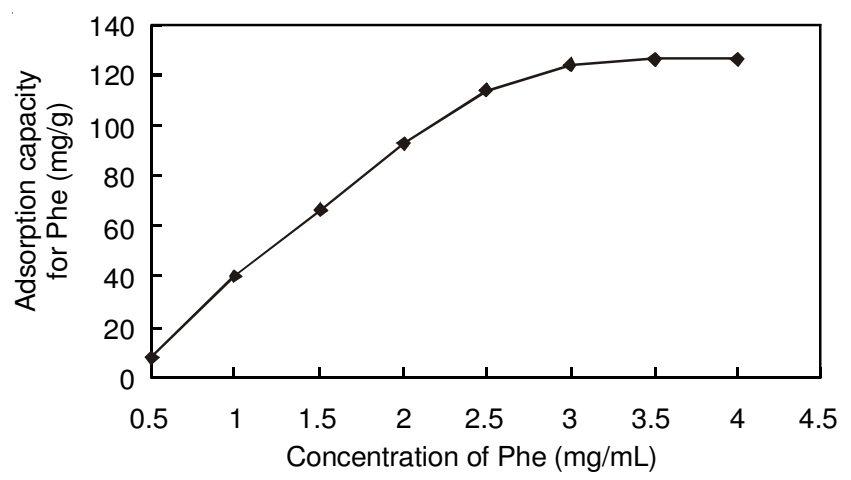

Fig. 3. Influence of L-phenylalanine concentration on the adsorption capacity

\section{Applications}

Adsorption: The adsorption curve of amino acids mixture on PCCR could be seen from Fig. 4. The appearance of leakage point of ala, glu, lys, L-phenylalanine were 14, 24, 8 and $41 \mathrm{~mL}$, respectively. The appearance of leakage point of L-phenylalanine was later than the other amino acids, which indicated that the adsorption capacity of L-phenylalanine on

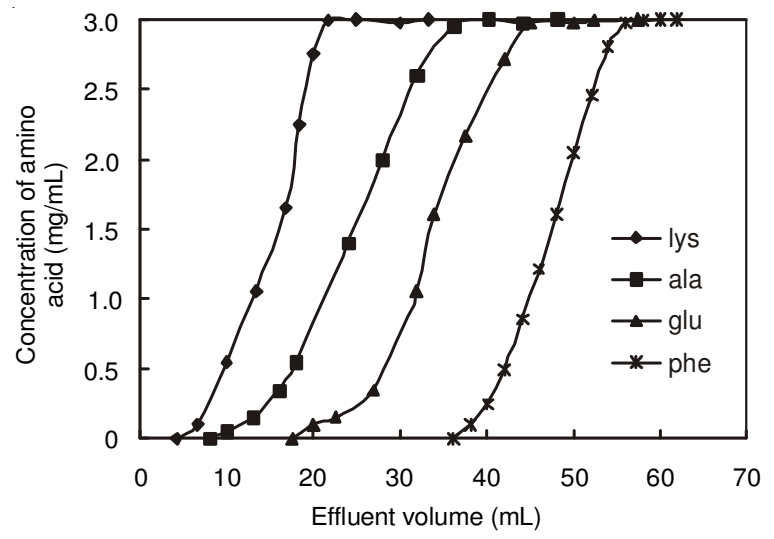

Fig. 4. Dynamic adsorption curve of amino acids mixture on PCCR

PCCR was higher. Calculated from the adsorption curve, the partition coefficient was, respectively $2.48,0.92,1.31,0.56$, which indicated that L-phenylalanine could be separated by PCCR from amino acids mixture.

Elution: The elution of L-phenylalanine and ala, glu, lys mixture was investigated and the elution curves were shown in Fig. 5. Ethanol and $\mathrm{NaOH}$ was taken to weaken the hydrophobic interaction and electrostatic interaction between Lphenylalanine and PCCR. So the elution agent was $30 \%$ ethanol contains $0.05 \mathrm{~mol} / \mathrm{L} \mathrm{NaOH}$. When the elution flow rate was $0.5 \mathrm{~mL} / \mathrm{min}$, the elution curves of ala, glu and lys were narrow and symmetric. Fig. 5 showed that the elution curves of ala, glu and lys were overlapped, completely. This result indicated that ala, glu and lys could not be separated effectively by PCCR. However, the curve of L-phenylalanine was far away from them and the overlapping area was small. The overlapping areas of L-phenylalanine and ala, glu, lys were, respectively 6,17 and $2 \%$. After the completely elution of ala, glu, lys, the pure L-phenylalanine could be collected. The recovery rates were $78 \%$. It indicated that a great deal of pure L-phenylalanine could be separated effectively from amino acids mixture by PCCR.

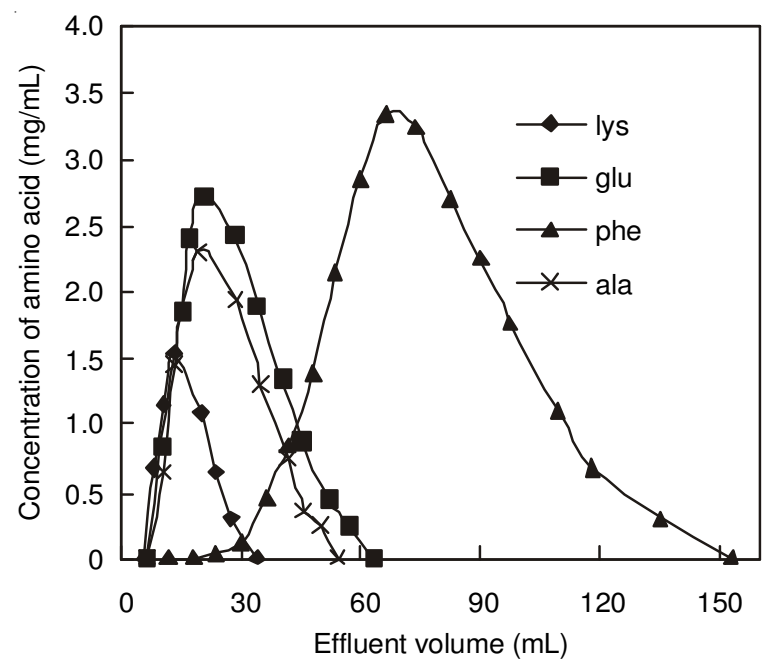

Fig. 5. Dynamic elution curve of L-phenylalanine, ala, glu and lys mixture

Stability: The repetitive stability of PCCR was showed in Fig. 6. After PCCR was cycled five times, the adsorption capacity of L-phenylalanine on PCCR was only decreased $4 \%$ 


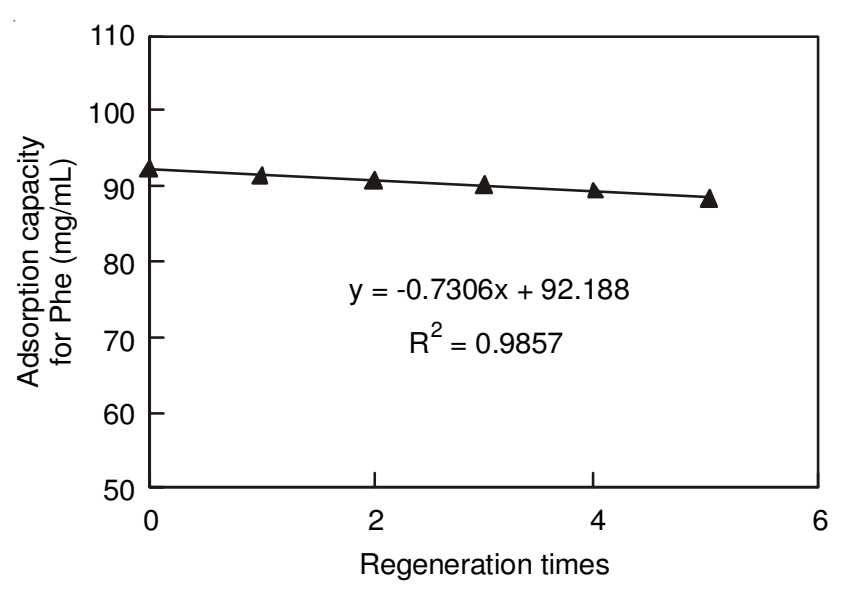

Fig. 6. Utilization stability of PCCR

and the resin was kept a good separation performance. The half-life of PCCR was about 64 times.

\section{Conclusion}

A macroporous anion exchanger with hydrophobic interaction resin L-phenylalanine-crosslinked chitosan resin (PCCR) was successfully prepared using semi-crosslinked chitosan and L-phenylalanine in this study. L-phenylalanine-crosslinked chitosan resin was successfully synthesized via the reaction between glutaraldehyde semi-crosslinked chitosan and Lphenylalanine. The introduction of flexible spacer arm improved the adsorption capacity of L-aromatic amino acid caused by $\pi-\pi$ hydrophobic interaction. The optimum adsorption condition was that the concentration was $3 \mathrm{mg} / \mathrm{mL}$, $\mathrm{pH}$ was 6 and flow rate was $3 \mathrm{~mL} / \mathrm{min}$. The amino acid could be eluted by $30 \%$ ethanol with $0.05 \mathrm{~mol} / \mathrm{L} \mathrm{NaOH}$ and flow rate was $0.5 \mathrm{~mL} / \mathrm{min}$. The recovery rate of pure L-phenylalanine was $78 \%$. The half-life of PCCR was about 64 times and kept a good stability. With remarkable performance for L-aromatic amino, PCCR obtained in this study possesses great potentials in many fields as a new kind of separation material.

\section{ACKNOWLEDGEMENTS}

This research was supported by the National Natural Science Foundation of China (Grant 21106191), Natural Science Foundation Project of CQ CSTC (Grant cstcjjA50002) and Fundamental Research Funds for the Central Universities (project No. CDJXS12220003, CDJXS10221138).

\section{REFERENCES}

1. M. Monier and A.M.A. El-Sokkary, Int. J. Biol. Macromol., 47, 207 (2010).

2. R.A. Kopper and R.P. Singhal, Int. J. Biol. Macromol., 1, 65 (1979).

3. M. Teramoto, T. Yamashiro, A. Inoue, A. Yamamoto, H. Matsuyama and Y. Miyake, J. Membr. Sci., 58, 11 (1991).

4. J.H. Wu, Z. Wang and S.Y. Xu, Food Chem., 103, 1255 (2007).

5. Y.S. Liu, Y.Y. Dai and J.D. Wang, Sep. Sci. Technol., 34, 2165 (1999).

6. A.S.K. Kumar, N. Rajesh, S. Kalidhasan and V. Rajesh, J. Environ. Sci. Health A, 46, 1598 (2011).

7. A. Ustinov, H. Weissman, E. Shirman, I. Pinkas, X. Zuo and B. Rybtchinski, J. Am. Chem. Soc., 133, 16201 (2011).

8. Y. Xiao, J. Wang and X.H. Zhou, Chin. J. Appl. Chem., 26, 780 (2009).

9. G. Singh, A.K. Sharma, A.K. Mishra and L. Prakash, Phosphorus Silicon Sulfur Rel. Elem., 133, 83 (1998).

10. P. Allard, L.D. Cowell, T.H. Zytkovicz, M.S. Korson and M.G. Ampola, Clin. Biochem., 37, 857 (2004).

11. Y.R. Tahboub and H.L. Pardue, Anal. Chim. Acta, 173, 23 (1985).

12. F. Wibrand, Clin. Chim. Acta, 347, 89 (2004).

13. X.H. Zhou, H.B. Zhao and S. Zhao, Sep. Sci. Technol., 46, 1977 (2011).

14. L.L. Fan, Y. Zhang, X.J. Li, C.N. Luo, F.G. Lu and H.M. Qiu, Colloid Surf. B, 91, 250 (2012).

15. G.T. Jia and X.Y. Lu, J. Chromatogr. A, 1193, 136 (2008).

16. Y. Fu, Y. Zu, W.Li, T. Efferth, N. Zhang, X. Liu and Y. Kong, J. Chromatogr. A, 1137, 145 (2006).

17. A.L. Lehninger, D.L. Nelson and M.M. Cox, Principles of Biochemistry, Worth Publishers, New York (1993).

18. A. Nicholls, K.A. Sharp and B. Honing, Protein Struct. Funct. Genet., 11, 281 (1991).

19. K.A. Sharp, A. Nicholls, R. Friedman and B. Honing, Biochemistry, 30, 9686 (1991). 